\title{
Dynamic Intervertebral Body Angle of the Lower Cervical Spine during Protracted Head Extension Using Measured by Fluoroscopy
}

\author{
Sung Hwa Seo, PT, PhD ${ }^{1,2)}$, Gak Hwang-Bo, PT, PhD ${ }^{3)}$, Goon-Chang Yuk, PT, PhD ${ }^{4}$, \\ Jin-Tae Han, PT, PhD ${ }^{5)}$, Joong-Hwi Kim, PT, PhD ${ }^{6}$, So Hyun Park, PT, PhD ${ }^{7}$ \\ 1) Department of Biomedical Sciences, Kyungpook National University \\ 2) Department of Medical Science, Gyeongju University \\ 3) Department of Physical Therapy, Daegu University \\ 4) Department of Physical Therapy, Yeungnam University Hospital \\ 5) Department of Physical Therapy, Kyungsung University \\ 6) Department of Physical Therapy, Catholic University of Daegu \\ 7) Department of Health Promotion, Daegu University: Jilyang, Gyeongsan, Gyeongbuk 712-714, \\ Republic of Korea.TEL: +82 53-850-5000,FAX: +82 53-850-5009,E-mail:ptpsh812@hanmail.net
}

\begin{abstract}
Purpose] To analyze dynamic lower cervical spine kinematics under neutral head extension (Ex) and protracted head extension (Pro-Ex) using fluoroscopy. [Method] The intervertebral body angle of the lower cervical spine of 8 healthy individual was analyzed using fluoroscopy during cervical extension with the head in the neutral (Ex) and protracted (Pro-Ex) positions. [Results] At maximum cervical extension position, we noted a significanly smaller value in the Pro-Ex position than in Ex position. During extension, the intervertebral body angle was significantly greater at C3-4 and less at C6-7 level in the Pro-Ex position compared to the Ex position in the initial phase. However, there was no significant difference in the intervertebral body angle between the two positions in final phase of extension. This shows greater extension movement C6-7 level in Pro-Ex. [Conclusion] The pro-Ex result showed less range of motion of extension. This suggests exaggerated hypermobility in the lower segments of the cervical spine, which might be implicated in early degenerative disease of the cervical spine.

Key words: Cervical dynamic kinematics, Fluoroscopy, Lower cervical spine
\end{abstract}

(This article was submitted Aug. 28, 2012, and was accepted Oct. 12, 2012)

\section{INTRODUCTION}

There is a growing incidence of neck and shoulder pain, owing to prolonged malalignment posture with head protraction $^{1,2)}$. It is usually caused by sedentary lifestyle, such as using a computer, watching TV or driving ${ }^{1,2)}$. Repetitive extension with head protraction can also alter the biomechanics of the cervical spine. These changes can induce severe neck pain or limitation of range of motion $(\mathrm{ROM})$, which can result in a vicious cycle of cervical disease $^{3-5)}$.

The evaluation of the cervical spine mobility of neck and shoulder pain patients is crucial for providing diagnostic and treatment information ${ }^{5-7)}$. Hypermobility and hypomobility are associated with degenerative change, pain, and dysfunctions such as reduced grip strength ${ }^{5-7)}$. Thus, segment motion analysis of the cervical spine is observed in a variety of fields. However, few studies have been conducted of dynamic motion of vertebral segments due to the complex movement of the spine ${ }^{8)}$ and the current limitations of dynamic analytical equipment in evaluation of each segment.

Motion capture systems and magnetic tracking devices are frequently used for dynamic motion analysis, but the markers attached to the skin can be significantly affected by skin movement resulting in high error rates ${ }^{9}$. Recently, fluoroscopy has been employed to analyze the dynamic kinematics of the spine ${ }^{10,11)}$. A fluoroscopy can be used to obtain high-quality dynamic motion images at low doses of radiation. However, cervical extension with the head in a protracted position has not yet been clearly elucidated.

In this study, we conducted fluoroscopic analysis of the cervical intervertebral body angle and lordosis of the lower cervical spine during cervical extension performed by healthy individuals with the head in the neutral extension (Ex) and protracted extension (Pro-Ex) positions. This is fundamental information for the evaluation and management of cervical disorders.

\section{SUBJECTS AND METHODS}

Eight subjects ( 8 females, mean age: $21.33 \pm 0.51$ years) with no history of neurologic or musculoskeletal disease involving the neck region participated in this experiment. The inclusion criteria were: age between 20-25 years, no subjective complaint of pain in the upper back, head, cheeks and upper limbs, no history of medical management for any 
spinal problem during the last year, and full ability to sit and stand without difficulty.

The exclusion criteria were: history of previous trauma to the cervical spine, currently undergoing medical treatment for neck pain, a diagnos is of systemic disease involving the cervical spine (e.g. rheumatoid arthritis), or pregnancy.

The subjects understood the principal objective of this study and provided their written informed consent before participating in the study. This protocol was approved by the Institutional Review Board of Yeungnam University Hospital, and was conducted in accordance with the ethical standards of the Declaration of Helsinki.

One trained physical therapist gave instructions to the subjects on the exact movements to perform. The subjects performed the cervical extension with the head in the Ex and Pro-Ex positions for 10-seconds. Neutral head extension (Ex) was a relaxed sitting posture with zero sagittal rotation and the face turned towards the ceiling. Protracted head extension (Pro-Ex), on the other hand, was the maximal forward gliding position with zero sagittal rotation and the face turned towards the ceiling as far as possible ${ }^{12)}$. A thoracolumbosacral orthosis (TLSO) was worn by the subjects to prevent compensatory movements of the lumbar and thoracic spine. The subjects practiced each position three times in preparation for the subsequent radiograph studies.

Video images were obtained with a fluoroscopy unit (ARCADIS Orbic, Siemens, USA) in the sagittal plane. Video data were acquired at 10 frames per second and subsequently converted to still images, resulting in approximately 100 images per cervical extension. However, approximately a quarter of these images were discarded because the image motion was blurred and the inferior end plate at $\mathrm{C} 7$ was occluded by the clavicle. Therefore, approximately only 25 images per cycle were used and these images were analyzed using LabVIEW software (National Instruments, USA).

The Cobb angle from $\mathrm{C} 3$ to $\mathrm{C} 7$ and intervertebral body angle were measured. The Cobb angle is the angle between the superior end plate of $\mathrm{C} 3$ and the inferior end plate of $\mathrm{C} 7$ and represents the lordosis of the lower cervical spine ${ }^{13)}$ (Fig. 1). The intervertebral body angle is the angle between the midplane of the adjacent vertebral spine as described by Frobin et al. ${ }^{14,15)}$ (Fig 1). The angle is counted positive, if the wedge opens ventrally. We determined that the Cobb angle was a reference value for comparison among the subjects, and the measured value was resampled to calculate the exact coordinates using a linear interpolation method. The interpolation was conducted using Matlab (Mathworks, USA). Differences between Ex and Pro-Ex were analyzed using the paired t-test. PASW 18.0 for Windows was used throughout. Statistical significance was accepted for values of $p<0.05$.

\section{RESULTS}

Eight subjects were enrolled in the study, all of who were females. They had a mean age of $21.33 \pm 0.51$ years, a mean weight of $51.83 \pm 3.37 \mathrm{~kg}$, and a mean height of $161.50 \pm 4.72 \mathrm{~cm}$.

In the comparison of Cobb angles between the Ex and Pro-Ex positions in the neutral cervical position, no

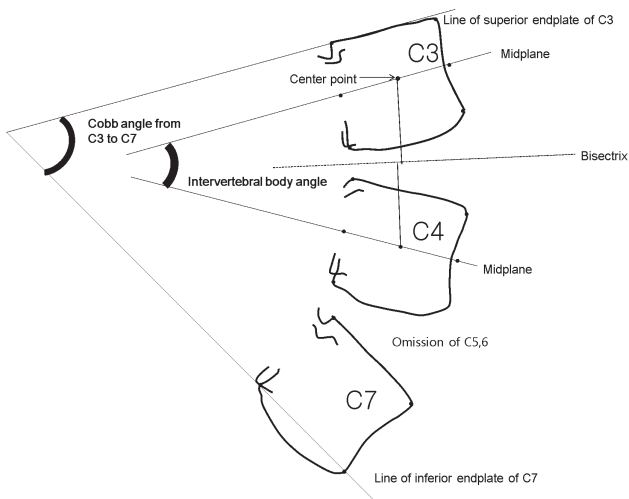

Fig. 1. Schematic diagram of the Cobb angle from $\mathrm{C} 3$ to $\mathrm{C} 7$ and the intervertebral body angle

significant difference was noted between the two positions $(p>0.05)$. However, at the maximum extension position, we noted a significantly smaller lordosis value in the Pro-Ex position than in the Ex position $(\mathrm{p}<0.05)($ Table 1).

The intervertebral body angle of each segment widened with increase in the Cobb angle (Table 2). The intervertebral body angle was less at the C3-4 level and greater at the C6-7 level in Pro-Ex than in Ex and there were a significant differences at Cobb angle of $15^{\circ}$ and $20^{\circ}(\mathrm{p}<0.05)$ (Table 2). However, there were no significant difference between two positions at Cobb angle of $25^{\circ}$ and $30^{\circ}$ (Table 2).

\section{DISCUSSION}

We analyzed the dynamic lordosis and intervertebral body angle of the lower cervical spine with fluoroscopy with the head in the neutral and protracted extension positions. We observed the kinematic changes of head position. To our knowledge, this is the first study to provide dynamic kinematic data according to head position changes during extension.

In maximal cervical extension, lordosis was less in the Pro-Ex position, which indicates that this position resulted in a smaller ROM of the lower cervical spine. Wu et al. ${ }^{11)}$ demonstrated that C5-6 has the highest intervertebral body angle during extension. Our results demonstrate that at maximum extension with the head protracted, C5-6 had the highest value but C6-7 had the smallest value and this may result in reduction in lordosis. Another possible reason for reduced lordosis in the Pro-Ex position is that the head is posteriorly located in Ex compared to Pro-Ex. This may result in greater lordosis in Ex than in Pro-Ex, because the posterior head weight may squeeze the lower cervical spine ${ }^{16}$. Kuo et al. ${ }^{17)}$ demonstrated that older adults generally have a forward head posture. The cervical extension range of motion was significantly reduced in this group compared to young adults. These results indicate those with chronic Visual Display Terminal syndrome or the elderly who have less lordosis of lower cervical spine may have a limited neck ROM.

With regards to intervertebral body angle, the upper segments, C3-4 and C4-5, demonstrated greater extension 
Table 1. Comparison of the Cobb angle from $\mathrm{C} 3$ to $\mathrm{C} 7$ between the Ex and Pro-Ex positions with neck upright and in maximum extension

\begin{tabular}{lcl}
\hline Cobb angle from C3 to C7 & $\begin{array}{c}\text { Ex } \\
\text { (Mean } \pm \text { SD) }\end{array}$ & $\begin{array}{c}\text { Pro-Ex } \\
\text { (Mean } \pm \text { SD) }\end{array}$ \\
\hline Upright Position & $7.12 \pm 4.52$ & $-0.06 \pm 7.9$ \\
Maximum Extension* & $39.34 \pm 5.5$ & $35.48 \pm 3.84$ \\
\hline
\end{tabular}

(unit: deg) The asterisk indicates the results of the paired t-test between Ex and Pro-Ex were significant $p<0.05$. The abbreviations used are as follows. Ex $=$ Neutral Extension position, Pro-Ex=Protracted Extension position

in the initial and middle phase in the Pro-Ex position and decreased ROM. The lower segment conversely appeared to show a compensatory motion in relation to this movement. Generally, degenerative changes frequently occur in the lower segment ${ }^{18)}$. The results of our study support the idea that the Pro-Ex position creates a higher ROM of intervertebral body angle at the C5-6 and C6-7 levels, possibly causing degenerative change in these segments during extension.

Finally, the trend in the intervertebral body angle demonstrated that extension of the upper segment was higher than that of the lower segment in the initial and middle phases during protraction. However, this trend changed in the final phase, at the Cobb angle of $30^{\circ}$. These findings suggest static or functional X-rays cannot represent dynamic kinematics, and dynamic kinematic analysis is needed to determine the exact causes of neck pain.

The limitations of this study were a small sample size and the lack of analysis of upper cervical motion due to the limited field of view available. The interpolation was used to identify trends of segmental motion.

In conclusion, the results suggest protraction is ineffective at increasing ROM and could exaggerate hypermobility of the lower cervical spine, which might be implicated in degeneration of the lower cervical spine. This should be helpful for the establishment and assessment of treatment strategies for patients with cervical impairment.

\section{REFERENCES}

1) Carter JB, Banister EW: Musculoskeletal problems in vdt work: a review. Ergonomics, 1994, 37: 1623-1648. [Medline] [CrossRef]

2) Iwakiri K, Mori I, Sotoyama M, et al.: VDT worker's posture and workload in free-address office system. Sangyo Eiseigaku Zasshi, 2006, 48: 7-14 [Medline] [CrossRef]

3) McPartland JM, Brodeur RR, Hallgren RC: Chronic neck pain, standing balance, and suboccipital muscle atrophy-a pilot study. J Manipulative Physiol Ther, 1997, 20: 24-29. [Medline]

4) Miyazaki M, Hymanson HJ, Morishita Y, et al.: Kinematic analysis of the relationship between sagittal alignment and disc degeneration in the cervical spine. Spine (Phila Pa 1976), 2008, 33: E870-876.

5) Rozen TD, Roth JM, Denenberg N: Cervical spine joint hypermobility: a possible predisposing factor for new daily persistent headache. Cephalalgia, 2006, 26: 1182-1185. [Medline] [CrossRef]

6) Bunketorp Käll L: Assessment of motion in the cervico-thoracic spine in patients with subacute whiplash-associated disorders. J Rehabil Med, 2008, 40: 418-425. [Medline] [CrossRef]

7) Cheng JS, Liu F, Komistek RD, et al.: Comparison of cervical spine kinematics using a fluoroscopic model for adjacent segment degeneration. Invited submission from the joint section on disorders of the spine and
Table 2. Comparison of the intervertebral body angles between the Ex and Pro-Ex positions at Cobb angle from $15^{\circ}$ to $30^{\circ}$

\begin{tabular}{llcc}
\hline $\begin{array}{c}\text { Cobb } \\
\text { angle }\end{array}$ & Segment & $\begin{array}{c}\text { Ex } \\
(\text { Mean } \pm \text { SE })\end{array}$ & $\begin{array}{c}\text { Pro-Ex } \\
(\text { Mean } \pm \text { SE })\end{array}$ \\
\hline $15^{\circ}$ & C3-4* & $2.13 \pm 1.38$ & $7.44 \pm 0.75$ \\
& C4-5 & $4.36 \pm 1.06$ & $5.98 \pm 1.57$ \\
& C5-6 & $5.50 \pm 1.40$ & $3.14 \pm 1.45$ \\
& C6-7* & $6.19 \pm 1.33$ & $0.93 \pm 0.99$ \\
\hline $20^{\circ}$ & C3-4* & $3.13 \pm 1.39$ & $7.84 \pm 1.53$ \\
& C4-5 & $6.07 \pm 1.3$ & $7.45 \pm 1.16$ \\
& C5-6 & $6.96 \pm 1.45$ & $4.99 \pm 1.69$ \\
& C6-7* & $6.74 \pm 1.08$ & $2.08 \pm 1.48$ \\
\hline $25^{\circ}$ & C3-4 & $4.78 \pm 1.07$ & $7.99 \pm 1.41$ \\
& C4-5 & $7.43 \pm 1.00$ & $7.93 \pm 1.24$ \\
& C5-6 & $7.70 \pm 1.13$ & $7.77 \pm 1.23$ \\
& C6-7 & $7.55 \pm 1.18$ & $4.43 \pm 1.59$ \\
\hline $30^{\circ}$ & C3-4 & $6.16 \pm 0.75$ & $8.28 \pm 1.06$ \\
& C4-5 & $8.41 \pm 0.92$ & $8.68 \pm 0.99$ \\
& C5-6 & $10.55 \pm 1.04$ & $10.09 \pm 1.05$ \\
& C6-7 & $7.52 \pm 1.36$ & $5.89 \pm 1.33$ \\
\hline
\end{tabular}

(unit: deg) The asterisk indicates the results of the paired t-test between Ex and Pro-Ex were significant $\mathrm{p}<0.05$. The abbreviations used are as follows. $E x=$ Neutral Extension position, Pro-Ex=Protracted Extension position

peripheral nerves, march 2007. J Neurosurg Spine, 2007, 7: 509-513. [Medline] [CrossRef]

8) Bogduk N, Mercer S: Biomechanics of the cervical spine. I: Normal kinematics. Clin Biomech (Bristol, Avon), 2000, 15: 633-648. [Medline] [CrossRef]

9) Koerhuis CL, Winters JC, van der Helm FC, et al.: Neck mobility measurement by means of the 'flock of birds' electromagnetic tracking system. Clin Biomech (Bristol, Avon), 2003, 18: 14-18. [Medline] [CrossRef]

10) Wu SK, Kuo LC, Lan HC, et al.: The quantitative measurements of the intervertebral angulation and translation during cervical flexion and extension. Eur Spine J, 2007, 16: 1435-1444. [Medline] [CrossRef]

11) Wu SK, Kuo LC, Lan HC, et al.: Segmental percentage contributions of cervical spine during different motion ranges of flexion and extension. J Spinal Disord Tech, 2010, 23: 278-284. [Medline] [CrossRef]

12) Ordway NR, Seymour RJ, Donelson RG, et al.: Cervical flexion, extension, protrusion, and retraction. A radiographic segmental analysis. Spine (Phila Pa 1976), 1999, 24: 240-247.

13) Bono CM, Vaccaro AR, Fehlings M, et al.: Measurement techniques for lower cervical spine injuries: Consensus statement of the spine trauma study group. Spine (Phila Pa 1976), 2006, 31: 603-609.

14) Frobin $W$, Brinckmann $P$, Biggemann $M$, et al: Precision measurement of disc height, vertebral height and sagittal plane displacement from lateral radiographic views of the lumbar spine. Clin Biomech (Bristol, Avon), 1997, 12: S1-S63. [Medline] [CrossRef]

15) Frobin W, Leivseth G, Biggemann M, et al.: Sagittal plane segmental motion of the cervical spine. A new precision measurement protocol and normal motion data of healthy adults. Clin Biomech (Bristol, Avon), 2002, 17: 21-31. [Medline] [CrossRef]

16) Takasaki H, Hall T, Kaneko S, et al.: A radiographic analysis of the influence of initial neck posture on cervical segmental movement at end-range extension in asymptomatic subjects. Man Ther, 2011, 16: 74-79. [Medline] [CrossRef]

17) Kuo YL, Tully EA, Galea MP: Video based measurement of sagittal range of spinal motion in young and older adults. Man Ther, 2009, 14: 618-622. [Medline] [CrossRef]

18) Lehto IJ, Tertti MO, Komu ME, et al.: Age-related mri changes at $0.1 \mathrm{t}$ in cervical discs in asymptomatic subjects. Neuroradiology, 1994, 36: 49-53. [Medline] [CrossRef] 